\title{
Is cognitive ability a factor in explaining differences in physiological and psychological stress responses?
}

Burcu Koksal

Department of Physiology, Lokman Hekim University, Çankaya, Ankara, Turkey

Submitted: 15 October 2021; Accepted: 19 January 2022

Online publication: 23 February 2022

Arch Med Sci 2022; 18 (2): 553-558

DOI: https://doi.org/10.5114/aoms/145940

Copyright @ 2022 Termedia \& Banach

\begin{abstract}
Introduction: Determining differences and patterns in responses might help to understand over-excitability of students with high cognitive ability (SHCA) and to design treatments for helping them in controlling stress.

Methods: A comparative research method was used with three different instruments. The data were analyzed by $t$-test and Welch's $F$-test.

Results: The SHCA reported significantly higher frequencies of psychological stress responses whereas they expressed significantly lower frequencies of physiological stress responses than their counterparts.

Conclusions: These findings might lead to a discussion about the factors determining differences in the responses given by students with high cognitive ability under stress conditions.
\end{abstract}

Key words: cognitive ability, psychological stress response, physiological stress response, stress.

Students with high cognitive ability (SHCA) experience heightened physiological and psychological experiences for different environmental stimuli. Moreover, their heightened experiences might lead to over-excitability. Five different forms of over-excitability of SHCA were determined: psycho-motor, sensual, intellectual, imaginational and emotional [1]. Piechowski and Cunningham defined psycho-motor over-excitability as heightened capacity for being physically active and intellectual over-excitability as heightened capacity and persistence for thinking and reasoning [2]. They defined sensual over-excitability as heightened pleasure for sensual experiences while they defined emotional over-excitability as heightened intensity of feeling and emotional awareness. The last one, imaginational over-excitability, is defined as heightened capacity to use images and metaphors and for living in world of fantasy and imagination.

Yakmaci-Guzel and Akarsu in their study compared individuals with high, median and low cognitive ability in terms of over-excitability [3]. Their findings revealed that high cognitive ability and over-excitability are associated and SHCA have more over-excitability than their counterparts with low and median cognitive abilities. Karpinski et al. saw this difference as indicating that SHCA are at risk for psychological and physiological disorders [4]. Based on their findings, the authors established a theory for explaining over-excitability. Their theory of hyper-brain/hyper-body explained that high cognitive capacity is a risk factor for psychological and physiological stress conditions such as anxiety, ADHD and mood dis-

\author{
Corresponding author: \\ Burcu Koksal PhD \\ Department of Physiology \\ Lokman Hekim University \\ Çankaya, Ankara, Turkey \\ Phone: +905069725038 \\ E-mail: burkirimhan@gmail. \\ com
}


orders. They studied 2213 male and 1472 female members of American MENSA, Ltd., a society of persons in the upper $2 \%$ of individuals taking an intelligence test. Their findings supported the idea that SHCA have a greater tendency to respond to stressors by ruminating and worrying, which are important predictors of psychological and physiological over-excitability leading to the disorders. The theoretical associations among over-excitability, stress and high cognitive ability can be represented by the following figure (Figure 1).

As seen from the model represented in Figure 1, two response types for over-excitability in stressful conditions are psychological and physiological responses. However, in the literature, perceived stress and general stress perceptions of SHCA have been studied without detailed data about psychological and physiological responses under specific stress conditions. Also, more studies have focused on the biological mechanism of stress responses of different subjects involving animals and ordinary or ill human subjects [5]. As one rare example focusing on individuals with high cognitive ability, Jung et al. investigated associations between high cognitive ability, stress and cytokines [6]. Their findings revealed that IQ levels of participants were positively associated with high levels of interferon $\gamma$ (IFN- $\gamma$ ) and interleukin-10 (IL-10) while tumor necrosis factor $\alpha$ (TNF- $\alpha$ ) and IL-6 were negatively associated with IQ levels. However, they did not find a direct relationship between intelligence and stress. Similarly, Singh and Sharma examined the relationship between perceived stress and stress reactivity, and general intelligence [7]. Their findings showed no relationship of general intelligence with perceived stress and stress reactivity. Sahin et al. also investigated the relationship between stress symptoms and cognitive ability by examining their experiences regarding stress symptoms in the past 6 months and cognitive ability scores [8]. They found no significant relationship between stress symptoms and cognitive abilities of the participants. However, the researchers looked at the stress phenomena from the responses of individuals; they saw details of the picture regarding the relationship between stress and cognitive ability. For example, Amini studied SHCA and compared them with their ordinary counterparts in terms of cognitive reactions towards stress stimuli [9]. He found that SHCA showed significantly more cognitive reactions to stress stimuli. Similarly, Bénony et al. also reported that SHCA exhibited more anxiety and hyperactivity as indications of reactions related to low academic self-esteem as a stress factor, compared to their peers [10]. Hence, it can be claimed that measurement of reactions towards stress by detecting physiological and psychological stress responses regarding different stressful conditions might provide a better way to explain differences in stress responses of SHCA and students with normal cognitive ability. Considering only experiences regarding the psychological side of the stress response in the last 6 months does not provide a good reflection of stress responses under different stressful conditions, because the response patterns of students should be examined in detail and general patterns should be determined to see the whole picture about stress responses of SHCA. Therefore, this study purposes to investigate the association of cognitive ability of middle school students with their physiological and psychological stress responses.

Methods. The method of the study was causal-comparative research and two different groups of healthy students were compared in terms of physiological and psychological stress responses. Based on the comparisons, differences in stress

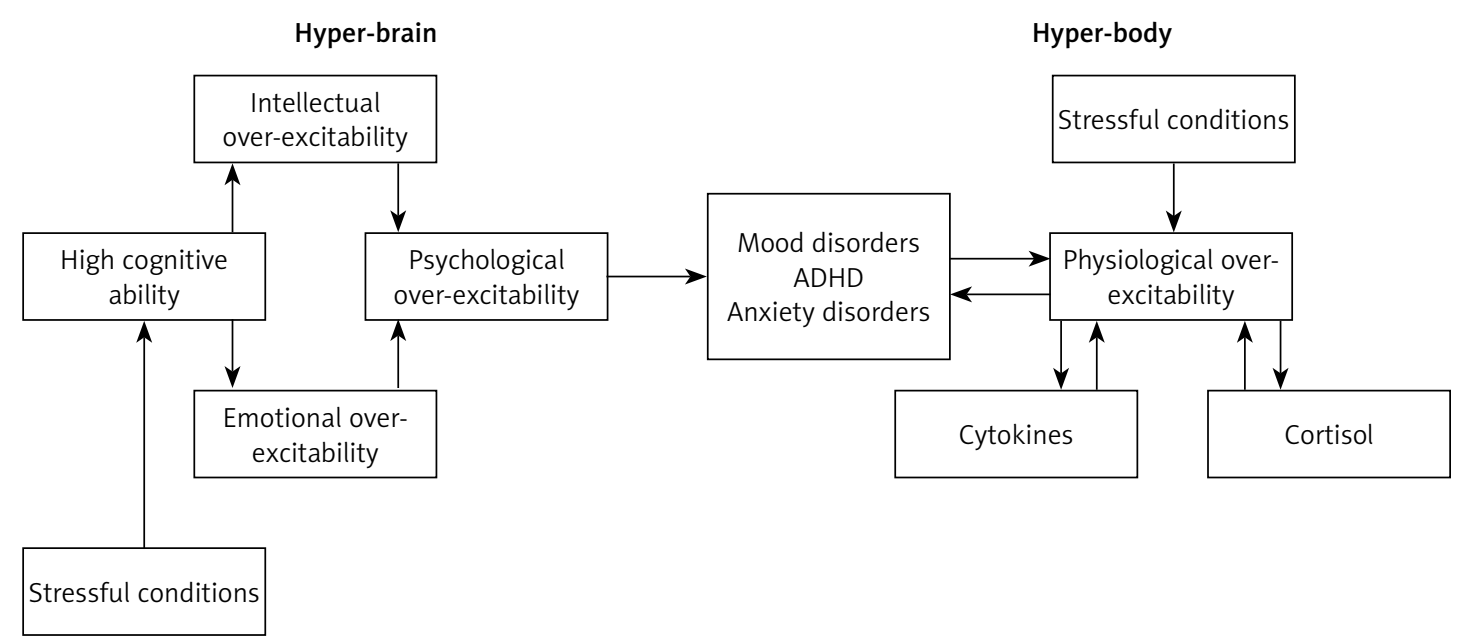

Figure 1. Associations among over-excitability, stress and high cognitive ability. The model is adapted from Karpinski et al. (2018) 
responses of the groups were investigated by calculating correlations.

Participants. Two hundred and thirty-five middle school students were involved in the study. The number of females $(n=123)$ was higher than the number of males $(n=112)$ in the sample. For sampling, convenient sampling was used to reach the participants in pandemic conditions. Fifty-seven participants were diagnosed as students with high cognitive ability and mean age of the participants was 11.65 . The majority of the participants (over 85\%) were doing physical exercises and regularly fed. Cognitive ability of the participants was determined according to their acceptance by a public gifted education program. If they were admitted to the program, we are sure that they had taken two different IQ tests (general cognitive ability test and Wechsler Non-Verbal Test) and they obtained scores over the cut-off of these standard tests. However, ordinary students might have taken the tests or not, but we know that a small number of them might have taken the tests. Therefore, we can compare them with students diagnosed as having high cognitive ability. We know that the scores of SHCA in this study were higher than the 130 IQ point as a formal cut-off score to enter a formal after-school gifted program for SHCA. Other students did not receive any diagnosis by such an application of an IQ test.

Instruments. In this study, three different instruments were used. The first one is a personal information form involving questions about age, gender, school type (high ability or ordinary), health problem, regularity in feeding and sportive activities. The second one is a questionnaire including 12 items asking about the frequency of different physiological responses under the stress condition. The items have four different options: 1 for never, 2 for sometimes, 3 for frequently and 4 for always. As an example for the items, "my blood pressure increases when I am under stress" is a kind of physiological response to stress conditions. The third one is another questionnaire with 15 items asking about the frequency of different psychological responses under the stress condition. The items of this instrument also have four different options: 1 for never, 2 for sometimes, 3 for frequently and 4 for always. As one example for the items, "I feel anxious when I am under stress" is a kind of psychological response to stress conditions. For validities and reliabilities of the scores on the questionnaires, Cronbach $\alpha$ calculation and confirmatory factor analysis was conducted. The results are presented in Table I.

As seen in Table I, the scores on the questionnaires have acceptable construct validity and reliabilities [11, 12].

Data analysis. For comparing the groups in terms of physiological and psychological stress responses, Welch's $F$ test was used. Also, mean and standard deviation were determined to describe data. At the same time correlations between physiological and psychological stress responses in different groups were also calculated.

Findings. Descriptive findings revealed that the students with high cognitive ability frequently present physiological stress responses under stress conditions (mean $=3.17(0.51)$ ). Similarly, the students with ordinary cognitive ability also frequently present physiological stress responses under stress conditions (mean $=2.93(0.82)$ ). In terms of psychological stress response, the students with high cognitive ability reported that they sometimes present this kind of response (mean = $1.53(0.37)$ ). The students with ordinary cognitive ability also indicated that they sometimes present this kind of response (mean $=1.70(0.52)$ ). The results of the comparisons showed that the students with high cognitive ability reported significantly higher frequencies of psychological stress responses than their ordinary counterparts (Welch's F $(1,154,34)=6.45, p<0.025)$. However, the students with high cognitive ability expressed significantly lower frequencies of physiological stress responses than their counterparts (Welch's $F(1,132,57)=7.16, p<0.025)$. In terms of paired comparisons, the students with high cognitive ability reported significantly higher frequencies for psychological stress responses than those for physiological stress responses (mean $_{\text {psy }}=3.17$, mean $_{\text {phy }}=1.53, t=14.82, \mathrm{~d} f=56, p<0.001$ ). Similarly, the students with ordinary cognitive ability reported significantly higher frequencies for psychological stress responses than those for physiological stress responses (mean $_{\text {psy }}=2.93$, $^{\text {mean }}$ phy $_{\text {. }}$ $=1.70, t=13.29, \mathrm{~d} f=56, p<0.001)$. When look-

Table I. Cronbach $\alpha$ and confirmatory factor analysis index values for the questionnaires

\begin{tabular}{|c|c|c|c|c|c|}
\hline \multirow[t]{2}{*}{ Instrument } & \multicolumn{4}{|c|}{ Confirmatory factor analysis index values } & \multirow[t]{2}{*}{ Cronbach $\alpha$} \\
\hline & $\mathrm{X}^{2} / \mathrm{d} f$ & CFI & GFI & RMSEA & \\
\hline $\begin{array}{l}\text { Questionnaire for } \\
\text { Physiological Stress } \\
\text { Responses }\end{array}$ & 2.64 & 0.88 & 0.90 & 0.08 & 0.84 \\
\hline $\begin{array}{l}\text { Questionnaire for } \\
\text { Psychological Stress } \\
\text { Responses }\end{array}$ & 2.44 & 0.94 & 0.88 & 0.08 & 0.94 \\
\hline
\end{tabular}


ing at the relationship between psychological and physiological stress responses across the groups, it is clear that physiological stress responses of the participants were negatively correlated with the psychological stress responses for both of the groups ( $r=-0.77, p<0.001$ for SHCA students; $r=-74, p<0.001$ for ordinary students). Distributions of the responses of the students in different groups for physiological and psychological stress responses are presented in Figure 2.

As seen in Figure 2, SHCA presented a narrower range of both physiological and psychological stress responses than their ordinary counterparts.

Discussion. The findings of this study revealed that SHCA reported significantly higher frequencies of psychological stress responses than their counterparts, whereas they expressed signifi- cantly lower frequencies of physiological stress responses than their counterparts. Also they reported a narrower range of physiological and psychological stress responses under different stress conditions. The findings are interesting in terms of reverse patterns in physiological and psychological stress responses. The biological mechanism of the stress response is well documented by previous animal and human studies [13-15]. However, this study reported more than possible biological mechanism by examining the psychological side of physiological and psychological stress responses of a special group of human subjects by directly asking them about their experiences. Hence the study reflects a different and important finding regarding stress experiences of SHCA as a sub-group of human subjects.
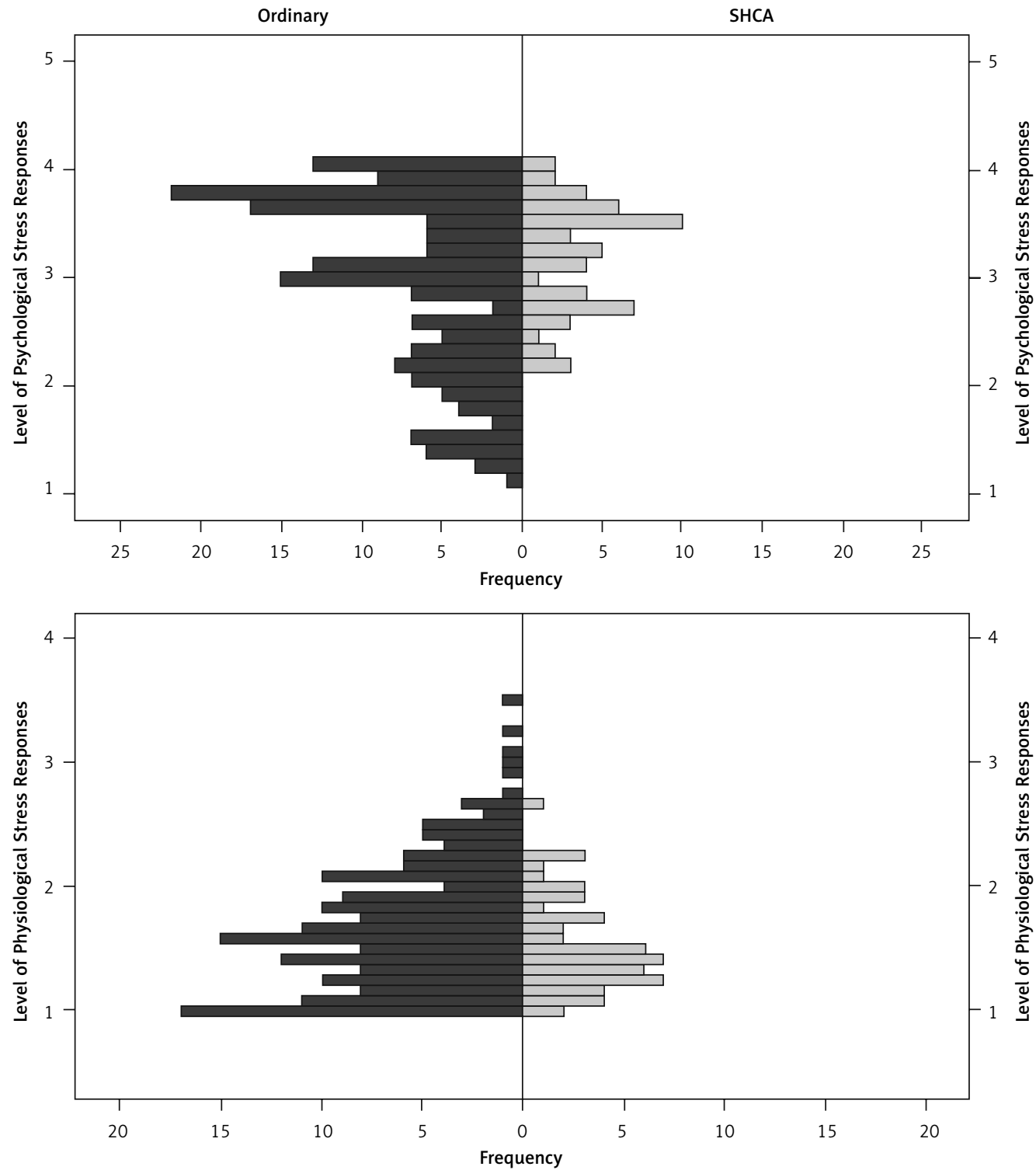

Figure 2. Distributions of the responses of the students in different groups for physiological and psychological stress responses (Note: Gray bars represent frequencies for SHCA while dark gray bars refer to frequencies for students with ordinary cognitive ability) 
By considering the findings of this study, we can claim that physiological and psychological stress responses do not go hand-in-hand when the SHCA is at the focus. Hence, new approaches to examine physiological and psychological stress responses are needed to see modeling changes in physiological and psychological responses after exposure to a stress condition. At the same time, the findings show the complex nature of examining physiological and psychological stress responses of SHCA, hence indicating a need to examine physiological and psychological stress responses of SHCA in a real context. The findings of this study might inform teachers and experts to design an effective learning environment for SHCA students and might also warn families with children with high cognitive ability about their inconsistency between different stress responses.

Previous studies focusing on general stress perceptions of SHCA found that SHCA experienced more stress than their ordinary counterparts [16, 17]. SHCA in this study also reported more stress than their ordinary counterparts when considering just psychological stress. Thus, we can claim that the disagreement with the literature might be related to the content of the instruments used in previous studies. Previous studies used a data collection instrument involving both physiological and psychological stress responses mixed in the same instrument [18]. But the stress response of individuals has two different sides: physiological and psychological stress responses. Hyper-brain and hyper-body theory reveals that the stress responses of SHCA should be examined from physiological and psychological aspects due to the over-excitability forms [4]. In line with this theory, this study also found different patterns in physiological and psychological stress responses of SHCA. Therefore, when the stress responses of SHCA are examined after collecting data from different instruments on physiological and psychological stress responses, different patterns and details might be seen. As in this study, stress responses of SHCA differed across different fields of stress responses when they were measured separately.

When the findings of this study are examined in detail, higher psychological and lower physiological stress responses of SHCA are not clinical and at serious levels for psychological disorders. As the reason for SHCA experiencing more psychological stress than ordinary counterparts, Webb stated that being SHCA, itself, is an important reason for psychological stress for SHCA [17]. Kaplan gave examples of different psychological stress factors regarding SHCA [18]. For example, peer pressure, struggle with labeling and parent pressure for high success are among the psychological stress factors [19]. Feld and Shusterman extended the list by adding high competition and pressure for maintaining high success [20]. These factors might increase psychological stress responses of SHCA. The psychological stress response is first seen and then the physiological stress response occurs [21]. But a lower level of physiological stress response might be provided by effective use of coping strategies after psychological experience of stress, since SHCA use psychological coping strategies more effectively than their ordinary counterparts [22]. This factor might decrease the level of the physiological stress response.

In conclusion, this study reported comparative findings about an important aspect of modern life: high cognitive ability and stress responses. However, there are some limitations regarding the sample and data analysis. First, the sample size is limited to 235 middle school students. It is necessary to increase the sample size to see changes in ranges of stress responses and to use more powerful statistical analysis. Second, the data analysis is limited to comparison of the groups by Welch's $F$ test; however, equality of the groups in terms of group members can be provided and additional variables such as severity of psychological experiences can be added to the analysis. At the same time, different groups of middle school students should be differently analyzed in terms of physiological and psychological stress responses in this study. For example, handicapped students might provide more detailed information.

\section{Conflict of interest}

The author declares no conflict of interest.

\section{References}

1. Ackerman CM. Identifying gifted adolescents using personality characteristics: Dabrowski's over-excitabilities. Roeper Rev 1997; 19: 229-36.

2. Piechowski MM, Cunningham K. Patterns of overexcitability in a group of artists. J Creat Behav 1985; 19: 153-74.

3. Yakmaci-Guzel B, Akarsu F. Comparing overexcitabilities of gifted and non-gifted 10th grade students in Turkey. High Abil Stud 2006; 17: 43-56.

4. Karpinski RI, Kolb AMK, Tetreault NA, et al. High intelligence: a risk factor for psychological and physiological over-excitabilities. Intelligence 2018; 66: 8-23.

5. Bove M, Fogacci F, Quattroccchi S, Veronesi M, Cicero AFG. Effect of Synaid on cognitive functions and mood in elderly subjects with self-perceived loss of memory after COVID-19 infection. Arch Med Sci 2021; 17: 1797-9.

6. Jung YH, Shin NY, Jang JH, et al. Relationships among stress, emotional intelligence, cognitive intelligence, and cytokines. Medicine 2019; 98: e15345.

7. Singh Y, Sharma R. Relationship between general intelligence, emotional intelligence, stress levels and stress reactivity. Ann Neurosci 2012; 19: 107-11.

8. Sahin NH, Guler M, Basim HN. The Relationship between cognitive intelligence, emotional intelligence, coping 
and stress symptoms in the context of type a personality pattern. Turk J Psychiatry 2009; 20: 243-54.

9. Amini M. Identifying stressors and reactions to stressors in gifted and non-gifted students. Int Educ J 2005; 6: $136-40$.

10. Bénony $H$, Van Der Elst D, Chahraoui K, et al. Link between depression and academic self-esteem in gifted children. Encephale 2007; 33: 11-20.

11. Kline R. Principles and practice of structural equation modeling. Guilford Press, New York 2011.

12. Schermelleh-Engel K, Moosbrugger $\mathrm{H}$. Evaluating the fit of structural equation models: tests of significance and descriptive goodness-of-fit measures. Methods of Psychological Research Online 2003; 8: 23-74.

13. Azline MFN, Qodriyah MS, Akmal MN, et al. In vivo effect of Piper sarmentosum methanolic extract on stress-induced gastric ulcers in rats. Arch Med Sci 2019; 15: 223-31.

14. Akıncı A, Esrefoglu M, Cetin A, et al. Melatonin is more effective than ascorbic acid and $\beta$-carotene in improvement of gastric mucosal damage induced by intensive stress. Arch Med Sci 2015; 11: 1129-36.

15. Janusek D, Kania M, Zaczek R. Evaluation of T-wave alternans in high-resolution ECG maps recorded during the stress test in patients after myocardial infarction. Arch Med Sci 2015; 11: 99-105.

16. Kaur AP. Stress, coping, self-efficacy, resilience and social support in university students. Int J Sci Res 2018; 6: 662-5

17. Webb JT. Nurturing social-emotional development of gifted children. In: International handbook of research and development of giftedness and talent. Heller K, Mönks FJ, Passow AH (eds.). Pergamon Press, Oxford, UK 1993; 525-38.

18. Erdem Ş, Baloğlu M. Gifted adolescents' stress sources, reactions against stress and comparison with general adolescent population. Inonu Univ J Fac Educ 2018; 19 : 598-609.

19. Kaplan LS. Helping gifted students with stress management. Flyer File on Gifted Students. Berger S (ed.). Council for Exceptional Children Digest, Reston VA 1990.

20. Feld LD, Shusterman A. Into the pressure cooker: student stress in college preparatory high schools. J Adolesc 2015; 41: 31-42.

21. Ursin H, Eriksen HR. The cognitive activation theory of stress. Psychoneuroendocrinology 2004; 29: 567-92.

22. Shaunessy E, Suldo SM. Strategies used by intellectually gifted students to cope with stress during their participation in a high school international baccalaureate program. Gift Child Q 2010; 54: 127-37. 